\title{
THE
}

\section{Theoretical Studies of the Structure and Dynamics of Metal/ Hydrogen Systems: Diffusion and Path Integral Monte Carlo Investigations of Nickel and Palladium Clusters}

B. Chen

M. A. Gomez

M. Sehl

J. D. Doll

David L. Freeman

University of Rhode Island, dfreeman@uri.edu

Follow this and additional works at: https://digitalcommons.uri.edu/chm_facpubs

Terms of Use

All rights reserved under copyright.

\section{Citation/Publisher Attribution}

Chen, B., Gomez, M. A., \& Freeman, D. L. (1996). Theoretical Studies of the Structure and Dynamics of Metal/Hydrogen Systems: Diffusion and Path Integral Monte Carlo Investigations of Nickel and Palladium Clusters. Journal of Chemical Physics, 105(21), 9686-9694. doi: 10.1063/1.472798

Available at: http://dx.doi.org/10.1063/1.472798

This Article is brought to you for free and open access by the Chemistry at DigitalCommons@URI. It has been accepted for inclusion in Chemistry Faculty Publications by an authorized administrator of DigitalCommons@URI. For more information, please contact digitalcommons-group@uri.edu. 


\title{
Theoretical studies of the structure and dynamics of metal/hydrogen systems: Diffusion and path integral Monte Carlo investigations of nickel and palladium clusters
}

\author{
B. Chen, M. A. Gomez, M. Sehl, and J. D. Doll \\ Department of Chemistry, Brown University, Providence, Rhode Island 02912 \\ David L. Freeman \\ Department of Chemistry, University of Rhode Island, Kingston, Rhode Island 02881
}

(Received 3 May 1996; accepted 28 June 1996)

\begin{abstract}
Using both classical and quantum mechanical Monte Carlo methods, a number of properties are investigated for a single hydrogen atom adsorbed on palladium and nickel clusters. In particular, the geometries, the preferred binding sites, site specific hydrogen normal mode frequencies, and finite temperature effects in clusters from two to ten metal atoms are examined. Our studies indicate that hydrogen is localized in the present systems. The preferred hydrogen binding sites are found to be tetrahedral in clusters with five or fewer metal atoms and octahedral for clusters of six to ten atoms. The exceptions to this rule are $\mathrm{Ni}_{9} \mathrm{H}$ and $\mathrm{Pd}_{9} \mathrm{H}$ for which the outside, threefold hollow and the inside tetrahedral sites are preferred, respectively. Hydrogen induced "reconstruction" of bare cluster geometries is seen in seven and ten-atom clusters. (C) 1996 American Institute of Physics. [S0021-9606(96)03337-5]
\end{abstract}

\section{INTRODUCTION}

Hydrogen/metal systems represent an interesting and important class of materials. Hydrogen's small mass and uniquely large isotopic variation, for example, give rise to a number of intrinsically quantum mechanical many-body phenomena. The unusual inverse isotope effects seen for hydrogen diffusion in palladium ${ }^{1,2}$ are examples of this point.

Understanding the structure and dynamics of these hydrogen/metal systems is central to obtaining a detailed understanding of a variety of technologically significant areas. Hydrogen storage, material embrittlement, fuel cell development, and catalysis are all topics in which hydrogen's interactions with transition metals play a vital role.

From a theoretical point of view, metal/hydrogen systems pose both special challenges and unique opportunities. On the challenge side of the ledger, the highly quantum mechanical nature of these sytems typically means that one must deal with the full complexity of a finite temperature, many-body, quantum problem. While classically based methods are of some use, their ultimate applicability is limited. On the other hand, hydrogen represents arguably the simplest adsorbate. It is, therefore, perhaps the most promising system for obtaining first principles, microscopic estimates of the fundamental interactions.

Within the broad class of metal/hydrogen systems, clusters are of particular importance. Their high surface to volume ratio makes them ideal candidates for catalytic applications. Since many applications involve hydrogen, an understanding of the properties of metal/hydrogen clusters is of special significance. Bulk studies have shown that the transport ${ }^{2}$ and vibrational spectroscopy ${ }^{3-5}$ of hydrogen are very sensitive to substrate structure. The wide variety of geometries exhibited by clusters thus offers a noteworthy opportunity to examine such effects.
The present paper is a computational study of metal/ hydrogen clusters. Our purpose is to examine a variety of properties of nickel and palladium systems ranging from two to ten-metal atoms. Chosen because they constitute interesting dynamical extremes in the bulk, ${ }^{2,3}$ these two fcc metals exhibit a variety of cluster geometries for different cluster sizes. Using a combination of classical and quantum Monte Carlo methods, we determine the geometries, preferred hydrogen binding sites, and site variation of hydrogen normal mode frequencies as a function of cluster size. All hydrogen isotopes are considered. Finally, path integral Monte Carlo methods are utilized to study finite temperature effects.

The embedded atom method (EAM) of Daw and Baskes ${ }^{6}$ is utilized throughout. While it is, no doubt, an incomplete description of the present cluster systems, using the same potential in the current work as in previous surface studies $^{2,3,5}$ enables comparison of hydrogen's behavior in cluster and surface environments. Although we have chosen not to use them, we note that there are more recent EAM parameterizations for the nickel and hydrogen systems. ${ }^{7,8} \mathrm{Fi}-$ nally, we emphasize that none of the methods in the present study depend on the details of the potential used. More complete estimates of the microscopic interactions can and will be used as they become available.

The organization of the rest of the paper is as follows: Section II briefly reviews the EAM method. Classical potential minima for bare metal and hydrogen containing metal clusters are reported in Section III. Quantum mechanical effects on the ground state structure and hydrogen binding are examined in Sections IV and V through the use of normal modes, zero-point energy analysis, and diffusion Monte Carlo methods, respectively. Section IV also considers the variation of harmonic estimates of the vibrational frequencies with cluster size and binding site. Finally, Section VI 


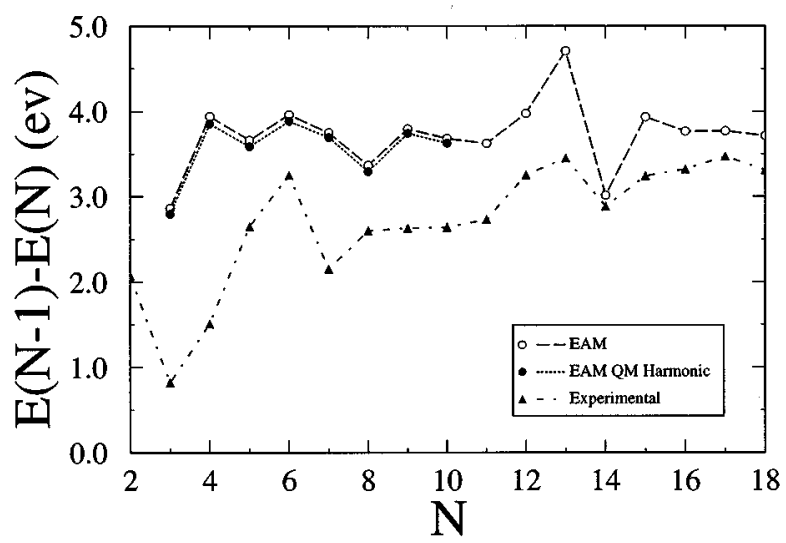

FIG. 1. Comparison of classical EAM data (Ref. 10) (open circles), zeropoint harmonic corrections to EAM data (solid circles), CEM (Ref. 11) (open triangles), and MD/MC-CEM (Ref. 11) (open diamonds) bare nickel cluster data with experimental atomic ejection data (Ref. 12) (solid triangles).

examines finite temperature effects using path integral Monte Carlo(PIMC).

\section{EMBEDDED ATOM METHOD}

In this section, we briefly review the EAM method. Details are discussed in the paper by Daw and Baskes. ${ }^{6}$ EAM expresses the total energy of the system as

$$
E_{\mathrm{tot}}=\sum_{i} F_{i}\left(\rho_{h, i}\right)+\frac{1}{2} \sum_{i \neq j} \frac{Z_{i}\left(r_{i j}\right) Z_{j}\left(r_{i j}\right)}{r_{i j}},
$$

where $F_{i}\left(\rho_{h, i}\right)$ is the embedding energy of atom $i$ in the host of the other atoms and $Z_{i}\left(r_{i j}\right)$ is the effective charge a distance $r_{i j}$ away from atom $i$. In the EAM formalism, the embedding energy depends on $\rho_{h, i}$, the electron density of the host at position $\mathbf{r}_{i}$ without atom $i$. The electron density of the host is approximated by the sum of atomic densities of the atoms in the host

$$
\rho_{h, i}\left(\mathbf{r}_{i}\right)=\sum_{j \neq i} \rho_{j}^{\text {atomic }}\left(r_{i j}\right) .
$$

A linear combination of $s$ and $d$ orbitals calculated by Clementi ${ }^{9}$ is used for the atomic densities of nickel and palladium. The embedding energy and effective charge are fit to experimental data for the bulk system.

Even though EAM is fit to bulk data, previous research shows that EAM is robust and yields at least qualitative data for the structure and binding energies of clusters. A measure of the quality of EAM potentials for nickel clusters is obtained by comparing its predictions of cluster structure and binding energies with those obtained by other methods. Such a comparison is given in Fig. 1 where sequential binding energies, $E(N-1)-E(N)$, are given as a function of cluster size, $N$, from a variety of methods. The open circles represent EAM results ${ }^{10}$ and the solid circles represent zero-point harmonic corrections to EAM values. Results obtained by Stave and DePristo ${ }^{11}$ (open triangles and diamonds) using computationally more intensive empirical potentials are also shown along with the experimental results of Lian et al. ${ }^{12}$ (solid triangles). The structures predicted by EAM methods ${ }^{10}$ are consistent with those inferred by Parks et al. ${ }^{13}$ from $\mathrm{N}_{2}$ absorption data with the exceptions of $\mathrm{Ni}_{8}$ and $\mathrm{Ni}_{14}$.

Although less extensive than the results for nickel systems, EAM methods ${ }^{14}$ have been applied to palladium clusters. Sachdev and Masel ${ }^{15}$ have examined palladium clusters ranging from 5 to 60 atoms. For magic-number-sized clusters, those with complete symmetry, the most stable structures are icosahedral. Non-magic number sized clusters have polyhedral structures. ${ }^{15}$ High resolution electron microscopy experiments on palladium clusters ${ }^{16}$ report icosahedral structures. It should be noted, however, that the experimental clusters are larger than those studied using the EAM potential.

\section{CLUSTER POTENTIAL ENERGY MINIMA}

We now turn to the issue of cluster structure. In so doing we will be interested in the geometries of clusters with and without hydrogen. Anticipating non-trivial quantummechanical effects, it is important to distinguish between structures predicted by purely classical-mechanical methods, those predicted by approximate, zero-point energy based quantum analyses, and the structures that emerge from numerically exact, diffusion Monte Carlo calculations. The present section focuses on classical results. Sections IV and $\mathrm{V}$ subsequently consider the corresponding quantummechanical results.

A simplex method ${ }^{17}$ is used to find the lowest potential energy structures of nickel and palladium clusters with 2-10 atoms with and without hydrogen. Between 1000 and 10000 clusters in random configurations are used as starting points for the simplex minimization. The lowest energy structures with and without hydrogen are compared. For reasons of space detailed cluster structures are not presented here, but are available from the authors upon request.

Examination of the global minimum geometries for nickel clusters with a hydrogen atom reveals stable binding sites in tetrahedral, octahedral, and three-fold hollow sites. In clusters not large enough to have octahedral sites, binding in tetrahedral sites occurs. Once there are sufficient atoms to form octahedral sites, that is at least six, there is a preference for octahedral sites. For reasons that will be discussed below, the exception to this is the nine-atom-nickel cluster where hydrogen binds on an outside, three-fold hollow site.

Comparing the global minimum geometries of the nickel clusters with a hydrogen atom to those without reveals rearrangements of the nickel clusters with the inclusion of a hydrogen atom. Figure 2 shows the global potential energy minima of nickel clusters with a hydrogen atom. In general, the geometry of the nickel clusters with and without the hydrogen is qualitatively the same. Bond lengths change upon addition of the hydrogen by only a few percent. The largest bond length change, about five percent, occurs for $\mathrm{Pd}_{7} . \mathrm{Ni}_{7}$ and $\mathrm{Ni}_{10}$, however, show significant changes upon addition of hydrogen. The global minimum potential energy structure of $\mathrm{Ni}_{7}$ without the hydrogen is pentagonal with an atom cap- 

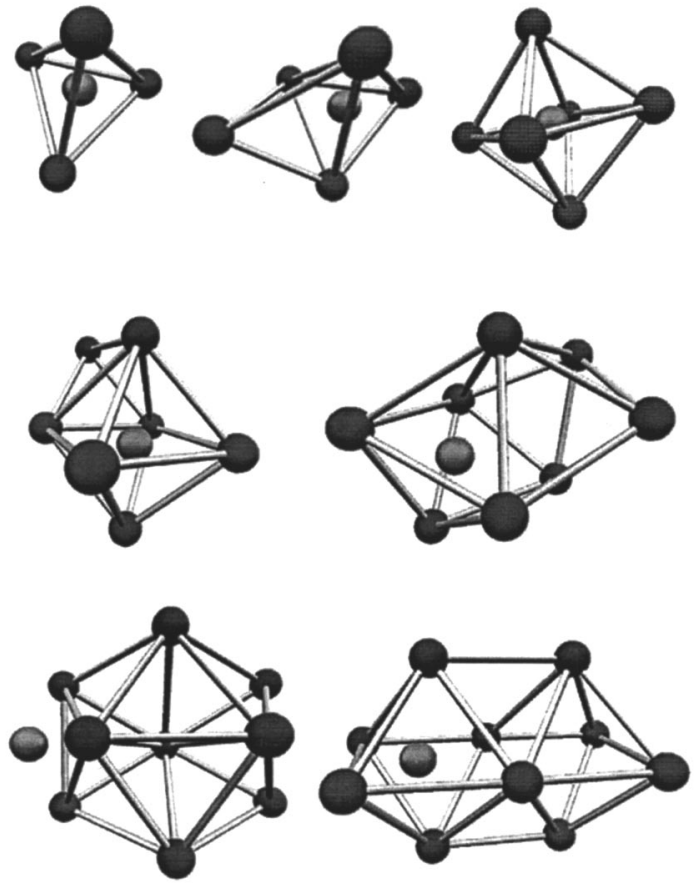

FIG. 2. Mininum energy structures of nickel clusters of sizes 4 to 10 atoms with a hydrogen atom. For purposes of identification, the $\mathrm{H}$ atom is shown without bonds to the nickel atoms.

ping both the top and bottom as seen in Fig. 3. The global minimum potential energy structure of the seven-atom-nickel cluster with the hydrogen has a structure incorporating a larger binding site for the hydrogen, namely an octahedral site. The preference of a larger hydrogen binding site is also seen in the ten-atom-nickel cluster as a comparison of Figs. 2 and 3 reveal. In both cases, where hydrogen induces a rearrangement of the cluster, the local minimum closest to the global minimum for the bare cluster has an octahedral site. These are examples of how the presence of a single hydrogen atom can lead to different substrate structures.

In general, the global minimum structures for nickel clusters with a hydrogen atom have the hydrogen bound in-
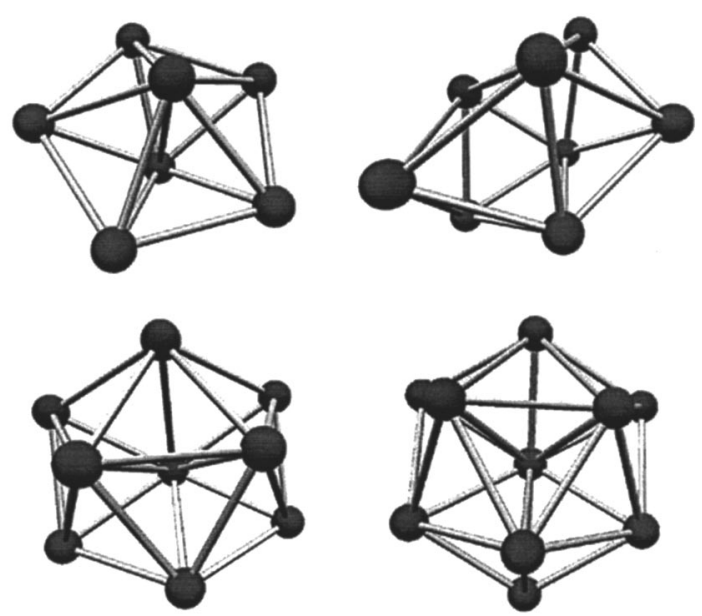

FIG. 3. Minimum energy structures for nickel clusters of sizes 7 to 10 atoms.
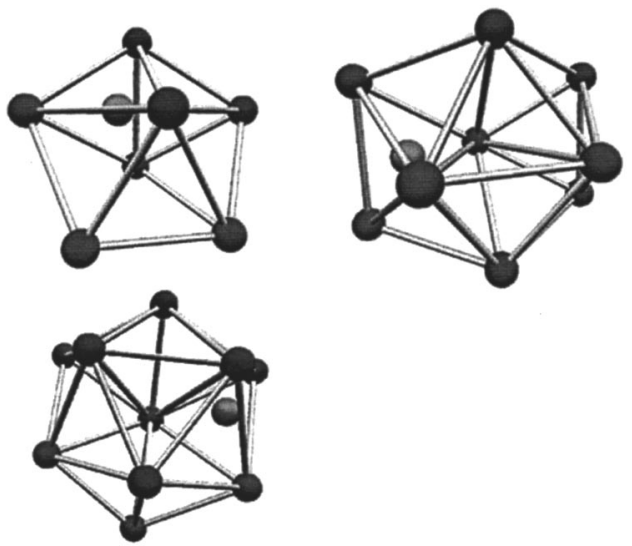

FIG. 4. Minimum energy structures for palladium clusters of sizes 7, 9, and 10 atoms with a hydrogen.

side the cluster. Once again the exception is $\mathrm{Ni}_{9} \mathrm{H}$. In this case, the energy for hydrogen binding inside a tetrahedral site in the cluster is $0.03 \mathrm{eV}$ higher than hydrogen binding outside the cluster. The most notable difference between the structure of $\mathrm{Ni}_{9} \mathrm{H}$ and the other structures in Fig. 2 is that enlarging a tetrahedral site to accommodate a hydrogen would compress adjacent tetrahedral sites. Using the present EAM potential, the preferred binding site of the hydrogen atom on the fcc (111) nickel surface is a three-fold hollow on the outside. ${ }^{3,6}$

The global potential energy minimum structures for palladium clusters with a hydrogen atom are the same as those for nickel clusters with the exceptions of $\mathrm{Pd}_{7} \mathrm{H}, \mathrm{Pd}_{9} \mathrm{H}$ and $\mathrm{Pd}_{10} \mathrm{H}$ which are shown in Figs. 4 and 5. In these cases, there
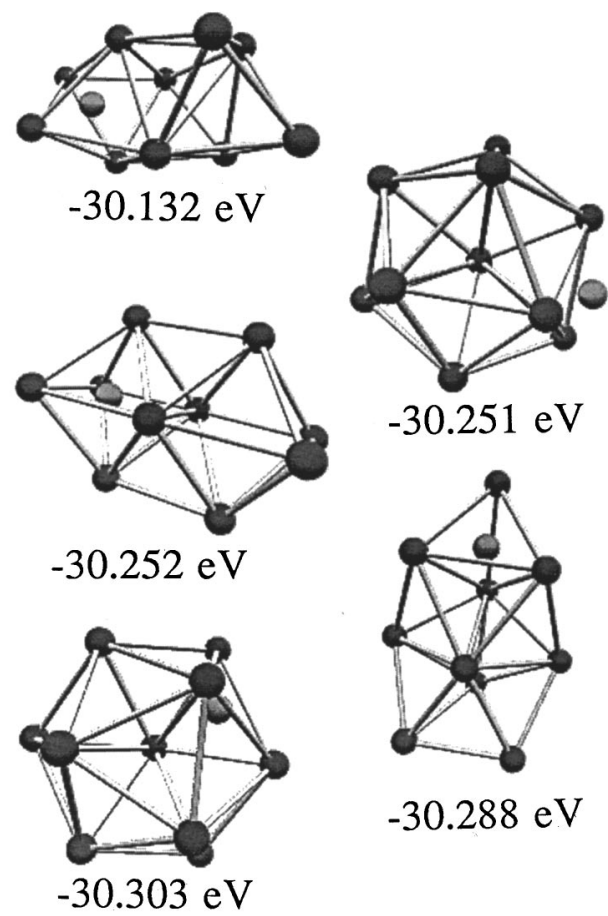

$-30.288 \mathrm{eV}$

FIG. 5. The five lowest potential energy isomers of $\mathrm{Pd}_{10} \mathrm{H}$ and their potential energies are shown. 


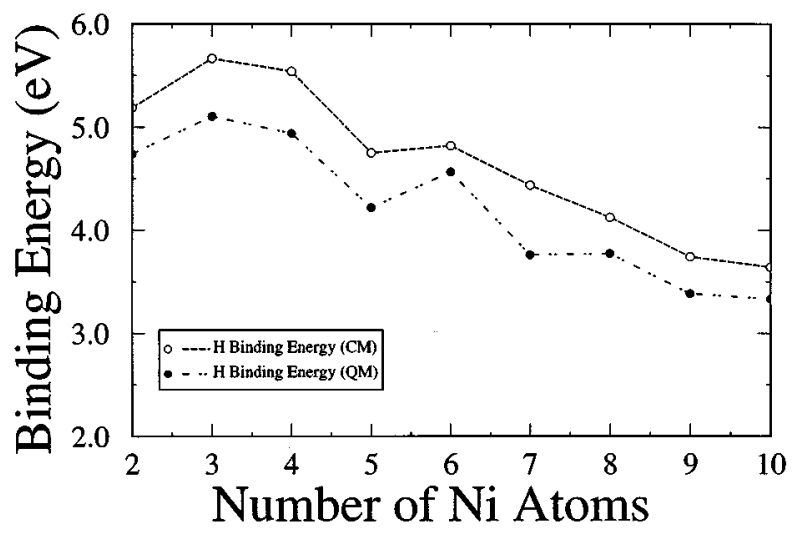

FIG. 6. Hydrogen binding energies of nickel clusters. Both classical and zero-point harmonic quantum corrections are shown. The classical hydrogen binding energies on (111), (100), and (110) fcc surfaces calculated with the same potential are $2.56 \mathrm{eV}, 2.66 \mathrm{eV}$, and $2.71 \mathrm{eV}$, respectively (Ref. 6).

is no rearrangement of the palladium atoms upon addition of the hydrogen to either the seven or ten-palladium-atom cluster. Further, for $\mathrm{Pd}_{9} \mathrm{H}$, the tetrahedral site is preferred over the outside site. This is not surprising since hydrogen readily percolates $^{18}$ through bulk palladium but has to be "pounded" into nickel. ${ }^{4}$ This suggests that palladium can accommodate hydrogen in its smaller binding sites far better than nickel can. The lattice constant in palladium is $3.89 \AA$ while in nickel it is $3.52 \AA$. The difference indicates that the lattice structure is more open in palladium than in nickel. A similar trend is seen in the clusters. The distances between hydrogen and the metal atoms defining a tetrahedral site are $1.467 \AA$ in $\mathrm{Ni}_{4} \mathrm{H}$ and $1.602 \AA$ in $\mathrm{Pd}_{4} \mathrm{H}$. The hydrogen-metal distances in an octahedral site are $1.662 \AA$ in $\mathrm{Ni}_{6} \mathrm{H}$ and 1.815 $\AA$ in $\mathrm{Pd}_{6} \mathrm{H}$.

Of the clusters under study, the one with a structure most similar to that of the (111) fcc surface is $\mathrm{Ni}_{7} \mathrm{H}$. This cluster is the smallest where both octahedral and tetrahedral sites are simultaneously present. The classical binding sites for the cluster, in order of lowest to highest energy, are octahedral, tetrahedral, and finally surface. This ordering is significantly different from that in the infinite surface modelled with the same potential. In the $\mathrm{Ni}(111)$ surface the corresponding classical ordering is surface site, octahedral site in the first subsurface layer, and lastly the tetrahedral site in the second subsurface layer. ${ }^{3}$ From the results it would appear that the seven-atom-cluster is able to expand to accommodate the hydrogen internally. As the cluster size increases, however, the presence of the surrounding atoms apparently makes such an expansion energetically less favorable and thus alters the site preference.

While these clusters are not sufficiently large to model an infinite surface, the binding energies are already beginning to approach those calculated for various infinite surfaces. ${ }^{3}$ Classical binding energies are found by subtracting the global minimum potential energy for the cluster with hydrogen from the global mininum for the cluster without hydrogen. Figure 6 shows the classical binding energies of hydrogen on the nickel cluster surfaces and the approach to

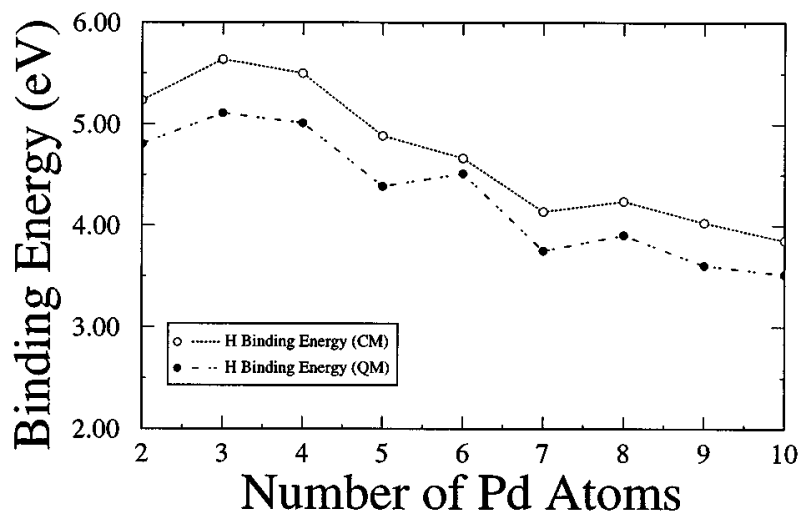

FIG. 7. Hydrogen binding energies of palladium clusters. Both classical and zero-point harmonic quantum corrections are shown. The classical hydrogen binding energies on (111), (100), and (110) fcc surfaces calculated with the same potential are $2.91 \mathrm{eV}, 2.91 \mathrm{eV}$, and $3.04 \mathrm{eV}$, respectively (Ref. 6).

the infinite surface values. The classical binding energies of hydrogen for the most stable binding sites on $\mathrm{Ni}(111)$, $\mathrm{Ni}(100)$, and $\mathrm{Ni}(110)$ fcc surfaces, calculated with the same potential, are $2.56 \mathrm{eV}, 2.66 \mathrm{eV}$, and $2.71 \mathrm{eV}$, respectively. ${ }^{6}$ For the ten-atom cluster, the binding energy for hydrogen is already $3.643 \mathrm{eV}$. The palladium clusters show a similar pattern in binding energies as Fig. 7 reveals. The classical binding energies of hydrogen for the most stable binding sites on the $\operatorname{Pd}(100), \operatorname{Pd}(111), \operatorname{Pd}(110)$ fcc surfaces are $2.91 \mathrm{eV}, 2.91$ $\mathrm{eV}$, and $3.04 \mathrm{eV}$, respectively. ${ }^{6}$ The ten-atom cluster is already at $3.852 \mathrm{eV}$.

\section{VIBRATIONAL FREQUENCIES}

It is useful to perform normal mode analyses for the low-lying stable cluster structures of the present systems. Such frequencies and the classical potential energy minima provide a convenient, zero-point energy approximation to the quantum-mechanical energies of the isomers. Because they reflect both the potential energy minima and its local curvature, the ordering of such zero-point estimates of isomer energies may differ from the corresponding classical ordering.

A summary of the harmonic estimates of the ground state of metal clusters with a hydrogen atom is given in Table II. In preparing these results harmonic estimates of the ground state energies of nickel and palladium clusters with and without hydrogen are calculated assuming the structures are those corresponding to the classical potential energy minima. When subsequent diffusion Monte Carlo (DMC) calculations described in the following section reveal that the quantum ground state geometry differs from the classical prediction, normal mode estimates are also prepared for the isomer corresponding to the DMC ground state structure.

We see in Table II that the ordering of the energies of the $\mathrm{Pd}_{7} \mathrm{H}$ and $\mathrm{Pd}_{10} \mathrm{H}$ isomers differ from classical predictions. For example, when modified by zero-point energy, the classical ground state structure of $\mathrm{Pd}_{10} \mathrm{H}$ shown in Fig. 5 with an energy of $-30.303 \mathrm{eV}$ moves up to an energy of $-29.566 \mathrm{eV}$ while the isomer predicted classically to have an energy of $-30.252 \mathrm{eV}$ becomes $-29.635 \mathrm{eV}$. We also 
TABLE I. Summary of normal mode vibrational frequencies for various sites. Surface theoretical frequencies are those found by Lynch et al. (Ref. $3)$. The frequencies in parentheses are experimental frequencies found by Ceyer et al. (Ref. 4). Parallel and perpendicular refer to the plane formed by the cluster atoms in the foreground of Fig. 8.

\begin{tabular}{lcc}
\hline \hline System & Parallel $\left(\mathrm{cm}^{-1}\right)$ & Perpendicular $\left(\mathrm{cm}^{-1}\right)$ \\
\hline $\mathrm{Ni}_{7} \mathrm{H}$ Cluster & & \\
$\quad$ Outside & 2601 & 1808 \\
$\quad$ Octahedral & 1643 & 1500 \\
$\quad$ Tetrahedral & 2848 & 3531 \\
Nickel Surface & & \\
$\quad$ Outside & $1360(955)$ & $1120(1170)$ \\
$\quad$ Subsurface Octahedral Site & $1137(800)$ & $893(800)$ \\
Pd ${ }_{7}$ H Cluster & & 1527 \\
$\quad$ Outside & 2650 & 1228 \\
$\quad$ Octahedral & 1591 & 3147 \\
$\quad$ Tetrahedral & 2445 & \\
Palladium Surface & & 945 \\
$\quad$ Outside & 1736 & 847 \\
$\quad$ Subsurface Octahedral Site & 1604 & \\
\hline \hline
\end{tabular}

note that the classical isomer shown in Fig. 5 with an energy of $-30.251 \mathrm{eV}$ produces a zero-point energy estimate of $-29.561 \mathrm{eV}$. Differences in zero-point energy thus break the near degeneracy of the classical $-30.252 \mathrm{eV}$ and -30.251 $\mathrm{eV}$ structures.

The calculated normal mode frequencies show patterns similar to those seen in the $\mathrm{Ni}(111)$ and $\mathrm{Pd}(111)$ surfaces. The sites in the quantum-mechanical $\mathrm{Ni}_{7} \mathrm{H}$ and $\mathrm{Pd}_{7} \mathrm{H}$ structures are analogous to those in the fcc(111) surface (cf. Fig. $8)$. The $C_{3 v}$ symmetry of the cluster's hydrogen environment mimics the symmetry of the localized surface binding site. Consequently, the symmetries of the hydrogen vibrational motion are the same for the cluster and the surface, namely a symmetric $\mathrm{A}_{1}$ state and a doubly degenerate $\mathrm{E}$ state. For $\mathrm{Ni}_{7} \mathrm{H}$ and $\mathrm{Pd}_{7} \mathrm{H}$ systems, the $\mathrm{Ni}-\mathrm{Ni}$ frequencies range from 105 to $674 \mathrm{~cm}^{-1}$ while the Pd-Pd frequencies range from 80 to $402 \mathrm{~cm}^{-1}$. Vibrational frequencies for the hydrogen atom in the clusters and various surface sites are summarized in Table I. Analysis of the normal mode eigenvectors for the cluster reveals that there are three modes to which the hydrogen atom contributes significantly. Two correspond to degenerate frequencies associated with "parallel" motion while one mode is associated with "perpendicular" displacements. Parallel and perpendicular are defined here relative to the plane of the three atoms in the foreground of Fig.

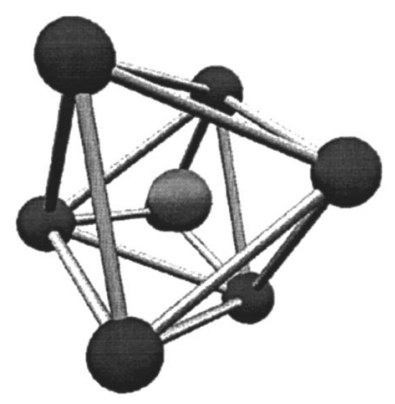

FIG. 8. $\mathrm{Ni}_{7} \mathrm{H}$ from a perspective that is reminiscent of the fcc (111) surface.
8. A comparison with surface data show that hydrogen vibrational frequencies on clusters are in general higher than those for hydrogen vibrating on the surface. Within a common EAM model, the seven-atom cluster and the (111) fcc surface exhibit the same type of behavior (i.e., the perpendicular vibration is of lower frequency than the parallel vibration except when the hydrogen occupies the tetrahedral sites).

From Table I we see that hydrogen's vibrational frequencies on surfaces and clusters decrease as one goes from an outside site to an interior octahedral site. This suggests that the geometry of the surroundings have a large impact on hydrogen's behavior. In discussing vibrational frequencies, however, it is important to keep a number of points in mind. First, the absolute quality of the predicted frequencies are a function of the quality of the underlying microscopic force law. More fundamentally, however, it may ultimately prove necessary to consider anharmonic effects in order to obtain an accurate description of the elementary excitations in the present systems.

\section{GROUND STATES}

While useful, the approximate quantum-mechanical treatments of Section IV are incomplete. It is therefore important to check the quality of the previous section's conclusions utilizing more rigorous methods. To that end we carry out diffusion Monte Carlo (DMC) ground state calculations for the present hydrogen/metal cluster systems. These we accomplish using importance sampling DMC techniques. Excellent reviews of the DMC method have been given elsewhere. ${ }^{19,20}$

The basis of the DMC method is that the Schrödinger equation when written in imaginary time $(\tau=\mathrm{it} / \hbar)$ is

$$
\frac{\partial \psi(\mathbf{r}, \tau)}{\partial \tau}=\left(\sum_{j} \frac{\hbar^{2}}{2 m_{j}} \nabla_{j}^{2}-\left(V(\mathbf{r})-E_{T}\right)\right) \psi(\mathbf{r}, \tau)
$$

where $m_{j}$ is the mass of particle $j, V(\mathbf{r})$ is the potential of the system, and $\psi(\mathbf{r}, \tau)$ is the wave function of the system. This is easily recognized as a diffusion equation with a growth/decay term. Solutions to this diffusion equation decay exponentially fast to the ground state. As a result, a simulation of diffusion and growth/decay processes for sufficiently long times yields ground state energies and wave functions. When importance sampling is included, the diffusion moves are biased in the direction of higher trial probability for the particle. Formally, the above equation is multiplied by trial wave function, $\phi_{T}(\mathbf{r})$. Rearrangement leads to

$$
\begin{aligned}
\frac{\partial f(\mathbf{r}, \tau)}{\partial \tau}= & \sum_{j} \frac{\hbar^{2}}{2 m_{j}} \nabla_{j}\left(\nabla_{j} f(\mathbf{r}, \tau)-f(\mathbf{r}, \tau) \nabla_{j} \ln \left|\phi_{T}(\mathbf{r})\right|^{2}\right) \\
& -\left(\frac{H \phi_{T}(\mathbf{r})}{\phi_{T}(\mathbf{r})}-E_{T}\right) f(\mathbf{r}, \tau)
\end{aligned}
$$

where

$$
f(\mathbf{r}, \tau)=\phi_{T}(\mathbf{r}) \psi(\mathbf{r}, \tau) .
$$


TABLE II. Summary of ground state energies for hydrogen with metal clusters. The minimum used for the harmonic estimates is that corresponding to the primary structure found in the DMC ground state. Usually, this corresponds to the classical global minimum except for $\mathrm{Pd}_{7} \mathrm{H}$ and $\mathrm{Pd}_{10} \mathrm{H}$. In these cases, the harmonic ground state found with the classical global minimum is in parenthess. The error bars given are one standard deviation.

\begin{tabular}{cccccc}
\hline \hline \multirow{2}{*}{$\begin{array}{c}\text { No. Metal } \\
\text { Atoms }\end{array}$} & \multicolumn{2}{c}{ Nickel $E_{0}(\mathrm{eV})$} & & \multicolumn{2}{c}{ Palladium $E_{0}(\mathrm{eV})$} \\
\cline { 2 - 3 } \cline { 5 - 6 } \cline { 5 - 6 } & Harmonic & DMC & & Harmonic & DMC \\
\hline 2 & -6.203 & $-6.222 \pm 0.001$ & & -6.135 & $-6.150 \pm 0.004$ \\
3 & -9.362 & $-9.40 \pm 0.01$ & & -8.948 & $-8.97 \pm 0.01$ \\
4 & -13.053 & $-13.04 \pm 0.01$ & & -12.237 & $-12.25 \pm 0.01$ \\
5 & -15.918 & $-15.928 \pm 0.002$ & & -14.716 & $-14.74 \pm 0.01$ \\
6 & -20.146 & $-20.113 \pm 0.001$ & & -18.193 & $-18.15 \pm 0.01$ \\
7 & -22.976 & $-23.02 \pm 0.01$ & & $-20.614(-20.576)$ & $-20.64 \pm 0.02$ \\
8 & -26.344 & $-26.34 \pm 0.01$ & & -23.624 & $-23.62 \pm 0.01$ \\
9 & -29.695 & $-29.71 \pm 0.02$ & & -26.571 & $-26.61 \pm 0.01$ \\
10 & -33.271 & $-33.22 \pm 0.04$ & & $-29.635(-29.566)$ & $-29.63 \pm 0.02$ \\
\hline \hline
\end{tabular}

This is a diffusion equation with a superimposed drift.

In the present work, we use a Gaussian trial wave function of the form

$$
\phi_{T}(\mathbf{r})=e^{-\frac{1}{2} \sum_{i<j} d_{i j}\left(r_{i j}-c_{i j}\right)^{2}}
$$

The values for $d_{i j}$ and $c_{i j}$ are found by minimizing the variance in the eigenenergy found by the trial wave function for configurations obtained using standard DMC. This is done for a cluster of two nickel atoms and a hydrogen. The resulting values for $d_{i j}$ and $c_{i j}$ are used in the trial wave function in the rest of the clusters. For physically reasonable choices, the ultimate results of the method are independent of the trial function used, although the efficiency of the approach varies with its choice.

Ground states are found for palladium and nickel clusters with a hydrogen atom. Results are summarized in Table II. All atoms are treated quantum mechanically. About 1000 walkers are used in the simulation. Initial walker configurations are generated by selecting replicas of approximately the ten lowest lying stable minima of the potential energy. Time steps ranging from $5 \times 10^{-7}$ ps to $5 \times 10^{-3}$ ps are used. The number of steps ranges from 10000 to 100000 . From 5 to 10 independent runs are done for each time step. While DMC is exact in the limit of zero time step, the ground state energy found using the smallest time step value is not always preferred. In some cases, the ground state energy at the smallest time step has a larger variance than that at larger time steps because of serial correlations. In practice, we use a time step that provides simultaneously a small variance with a ground state energy that does not differ significantly from that found at smaller time steps.

Ground states are not found for all bare clusters since a harmonic approximation to the ground state energy represents these adequately. For example, the DMC ground state energies for $\mathrm{Ni}_{7}$ and $\mathrm{Pd}_{7}$ are $-19.26 \pm 0.03 \mathrm{eV}$ and $-16.88 \pm 0.01 \mathrm{eV}$, respectively. The ground state energies using a harmonic approximation are $-19.277 \mathrm{eV}$ and $-16.864 \mathrm{eV}$.
Our ground state studies indicate that hydrogen in these systems is localized. This agrees with the findings of Rick and Doll on the Pd (111) fcc surface modeled with EAM interactions. ${ }^{5}$

The ground state structures of the clusters generally reflect the minimum potential energy structures. The exceptions are $\mathrm{Pd}_{7} \mathrm{H}$ and $\mathrm{Pd}_{10} \mathrm{H}$ systems. To gain insight into the details of these systems it is convenient to analyze the corresponding DMC ground state wave functions. In general, for an $N$-atom system the wave functions depend on $3 N$ spacial variables. In order to portray this high-dimensional object, we have adopted the following procedure: We begin by noting that the DMC calculation consists of Markoff sequences of "walkers" in this $3 N$ dimensional space. Each walker is a composite mathematical "cluster" in which the positions of the "atoms" correspond to those in physical systems. The DMC algorithm provides a set of stochastic rules by which the walker is advanced. It is important to recall that the walker configurations are representative of $\mathrm{f}(\mathbf{r}, \tau)$. As $\tau$ becomes large, $\mathrm{f}(\mathbf{r}, \tau)$ approaches $\phi_{0}(\mathbf{r}) \phi_{T}(\mathbf{r})$. Weighting each DMC configuration by $1 /\left[\phi_{T}(\mathbf{r})\right]$ thus produces an object that is representative of $\phi_{0}(\mathbf{r})$. In order to visualize the resulting configurations, it is convenient to divide three dimensional space into cells and to accumulate for the $n$th walker configuration, $\mathbf{r}_{n}$, a value of $1 /\left[\phi_{T}\left(\mathbf{r}_{n}\right)\right]$ in the cells corresponding to the position of each "atom" of the DMC walker. The center of mass for the walker remains at rest during the calculation. Provided that the cluster does not rotate appreciably during the calculation (a condition that is met in the present work), isosurfaces of the resulting histogram provide a sense of the ground state wave function. Using similar procedures while constraining various atoms to be in determined positions would allow us to examine various projections of the many-body ground state. Representative isosurfaces of $\phi_{0}(\mathbf{r})$ are shown in Fig. 9 for $\mathrm{Pd}_{7} \mathrm{H}$ and $\mathrm{Pd}_{10} \mathrm{H}$. The observed structures of these clusters are similar to the structures of $\mathrm{Ni}_{7} \mathrm{H}$ and $\mathrm{Ni}_{10} \mathrm{H}$. The minimum potential energy structures for these systems are shown in Fig. 2. The quantum mechanical character of the hydrogen in these clusters seems to induce a preference for a larger binding site. In 

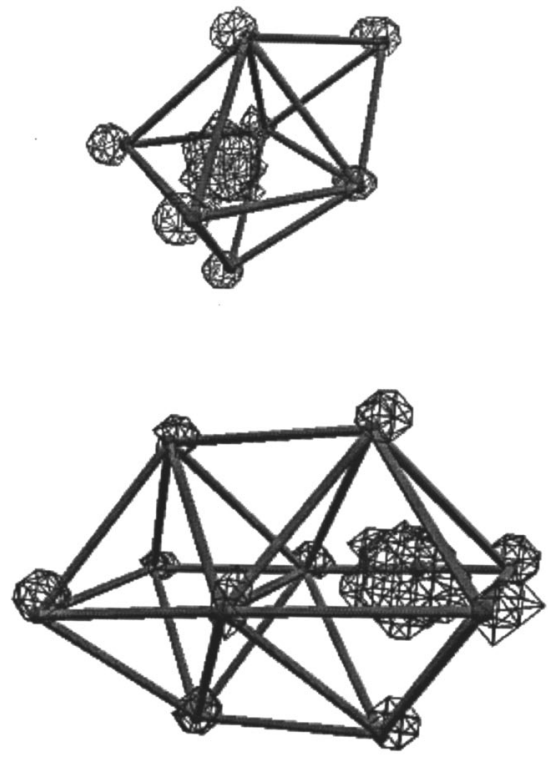

FIG. 9. $\mathrm{Pd}_{7} \mathrm{H}$ and $\mathrm{Pd}_{10} \mathrm{H}$ DMC ground state structures. One iso-surface of the DMC distribution of the atoms is shown. $\operatorname{In~} \mathrm{Pd}_{10} \mathrm{H}$ both octahedral sites are equivalent. In the simulation, hydrogen is started out at one of the octahedral sites. In the time of the calculation, hydrogen does not diffuse to the other octahedral site. The adiabatic potential energy barrier for hydrogen to diffuse from one octahedral site to the other is $1.336 \mathrm{eV}$.

each case, the binding site is octahedral. If hydrogen is replaced by deuterium in the seven-atom cluster, then the preferred binding site reverts to the global minimum energy classical structure. The ground state energies of $\mathrm{Pd}_{7} \mathrm{H}$, $\mathrm{Pd}_{7} \mathrm{D}$, and $\mathrm{Pd}_{7} \mathrm{~T}$ are $-20.64 \pm 0.02 \mathrm{eV},-20.71 \pm 0.02 \mathrm{eV}$, and $-20.76 \pm 0.02 \mathrm{eV}$, respectively.

Finally, we examine the ground states for $\mathrm{Pd}_{10} \mathrm{H}$, $\mathrm{Pd}_{10} \mathrm{D}$, and $\mathrm{Pd}_{10} \mathrm{~T}$. The normal mode based, zero-point energy analysis of Section IV suggests that quantummechanical effects are important in determining the structure of this system. Using DMC methods, it is possible to check these predictions.

The DMC ground states for $\operatorname{Pd}_{10} \mathrm{H}, \mathrm{Pd}_{10} \mathrm{D}$, and $\mathrm{Pd}_{10} \mathrm{~T}$ have the same dominant structure, namely that of the third lowest isomer shown in Fig. 5. An interpretation of the ground state is shown in Fig. 9. The ground state energies of $\mathrm{Pd}_{10} \mathrm{H}, \quad \mathrm{Pd}_{10} \mathrm{D}$, and $\mathrm{Pd}_{10} \mathrm{~T}$ are $-29.63 \pm 0.02 \mathrm{eV}$, $-29.67 \pm 0.03 \mathrm{eV}$, and $-29.73 \pm 0.02 \mathrm{eV}$, respectively.

\section{FINITE TEMPERATURE ENERGY AVERAGES}

The finite temperature energy averages are evaluated with the Fourier path integral method, in which the physical properties are obtained from the thermal density matrix $\rho\left(\mathbf{x}, \mathbf{x}^{\prime}, \beta\right)$. For a detailed description of the method, see Ref. 21 . Here we apply the temperature differentiation method ( $T$ method) to calculate the total energy. The $\mathrm{T}$ method utilizes the statistical mechanical expression

$$
\langle E\rangle=-(\partial \ln Q / \partial \beta)_{V, N},
$$

where the canonical partition function $Q$ is

$$
Q(T, V, N)=\int d \mathbf{x} \rho(\mathbf{x}, \mathbf{x}, \beta) .
$$

Using the expression for $\rho\left(\mathbf{x}, \mathbf{x}^{\prime}, \beta\right)$ (Ref. 21) derived from the Fourier path integral method, one gets the total energy for $N$ quantum particles, with finite number of Fourier coefficients $k_{\max }$, as follows

$$
\langle E\rangle_{k_{\max }}=\left\langle\overline{V_{a}}\right\rangle+\frac{1}{2 \beta}\left[3 N\left(k_{\max }+1\right)-\left\langle\sum_{k=1}^{k_{\max }} a_{k}^{2} / \sigma_{k}^{2}\right\rangle\right],
$$

where $\overline{V_{a}}$ is the average of the potential over the Fourier path, and the average terms $\langle\cdots\rangle$ are over the density $\rho\left(\mathbf{x}, \mathbf{x}^{\prime}, \beta\right)$ with respect to the space coordinate $\mathbf{x}$ and Fourier coefficients $\mathbf{a}_{k}$. Equal-spaced trapezoidal rule quadrature is used for the potential integral over the path. The $k_{\max }$ required for convergence increases as the system becomes increasingly quantum mechanical, as does the corresponding computational effort.

To speed up the convergence with respect to the number of included Fourier coefficients, a partial averaging method is applied to the calculation. A detailed description of the physical idea behind the partial averaging can be found in Ref. 21. With partial averaging the resulting energy expression is

$$
\begin{aligned}
\langle E\rangle= & \left\langle\overline{V_{a}}\right\rangle+\frac{1}{2 \beta}\left[3 N\left(k_{\max }+1\right)-\left\langle\sum_{k=1}^{k_{\max }} a_{k}^{2} / \sigma_{k}^{2}\right\rangle\right] \\
& +2\left[\left\langle\overline{V_{\mathrm{eff}}}\right\rangle-\left\langle\overline{V_{a}}\right\rangle\right],
\end{aligned}
$$

where $\overline{V_{\text {eff }}}$ is the average of the Gaussian transform of the system potential over the Fourier path. The last term is present only when the partial averaging is being utilized. Gradient partial averaging methods described in Ref. 21 are used in the present work.

When the path collapses to a single point, that is, the quadrature point for the potential integration is set to one, quantum effects go to zero. The energy expression reduces to the classical result

$$
\langle E\rangle=\langle V\rangle+\frac{3 N}{2 \beta} .
$$

With the above formalism, we calculate the total energy of the $\mathrm{Ni}_{\mathrm{n}} \mathrm{H}$ and $\mathrm{Pd}_{\mathrm{n}} \mathrm{H}$ clusters utilizing EAM. Here $k_{\max }$ ranges from 4 to 40 as temperature varies from $1060 \mathrm{~K}$ to 60 $\mathrm{K}$. Test runs show that the quantum contribution of the metal atoms (M) increases with increasing cluster size, from $0.3 \%$ at $\mathrm{M}_{2} \mathrm{H}$ to about $1 \%$ at $\mathrm{M}_{7} \mathrm{H}$. All the atoms are treated quantum mechanically in the calculation.

Sampling procedures are examined in the present work to determine whether or not configurations representative of different structured isomers are included. For example, the Monte Carlo configuration is quenched every 1200 moves via simulated annealing techniques. At temperatures above $500 \mathrm{~K}$, transitions between structured isomers are observed. Below $500 \mathrm{~K}$, the initial isomer is seen to persist throughout the entire one million Monte Carlo move run. 


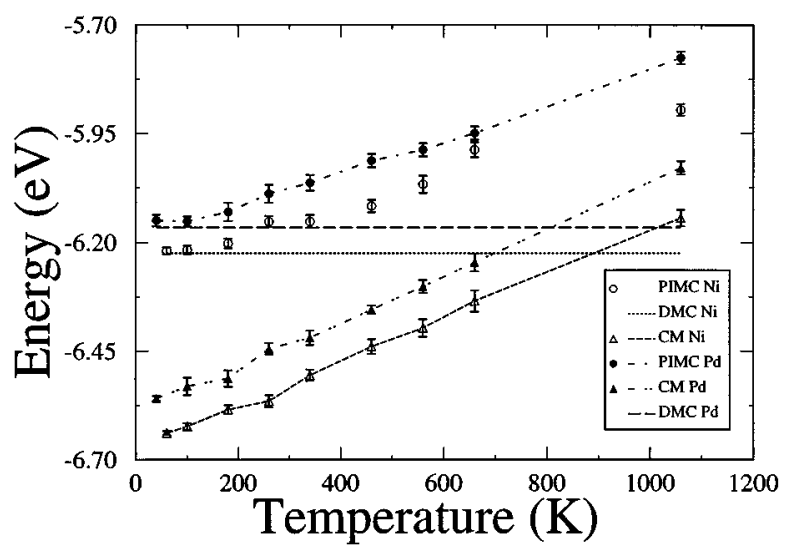

FIG. 10. $\mathrm{Ni}_{2} \mathrm{H}$ and $\mathrm{Pd}_{2} \mathrm{H}$ average energies as a function of temperature: open circle, open triangle and dotted line are the results of PIMC, CM and DMC respectively for the $\mathrm{Ni}_{2} \mathrm{H}$ cluster. Solid circle, solid triangle and dashed line are the results of PIMC, CM and DMC, respectively, for the $\mathrm{Pd}_{2} \mathrm{H}$ cluster.

Average energies are calculated as a function of temperature for different clusters for temperatures above $60 \mathrm{~K}$. The total Monte Carlo moves for each energy data point is about 400000 . The error bar shown is one standard deviation. The results are compared with the ground state energy from DMC and the classical limit. Both quantum and isotopic effects are investigated. We use the global potential energy minimum as the initial configuration in the energy calculation.

The calculated PIMC results agree with the corresponding DMC results, as can be seen from Figs. 10 to 12. The PIMC energies approach the ground state energy as the temperature decreases. The finite temperature classical results also approach the minimum potential energy of the cluster as the temperature decreases. Among the clusters investigated, $\mathrm{Ni}_{2} \mathrm{H}$ and $\mathrm{Pd}_{2} \mathrm{H}$ are most quantum mechanical. This is confirmed by the results shown in Fig. 10. The calculated curve appears to be flat at temperatures below $100 \mathrm{~K}$ because of the

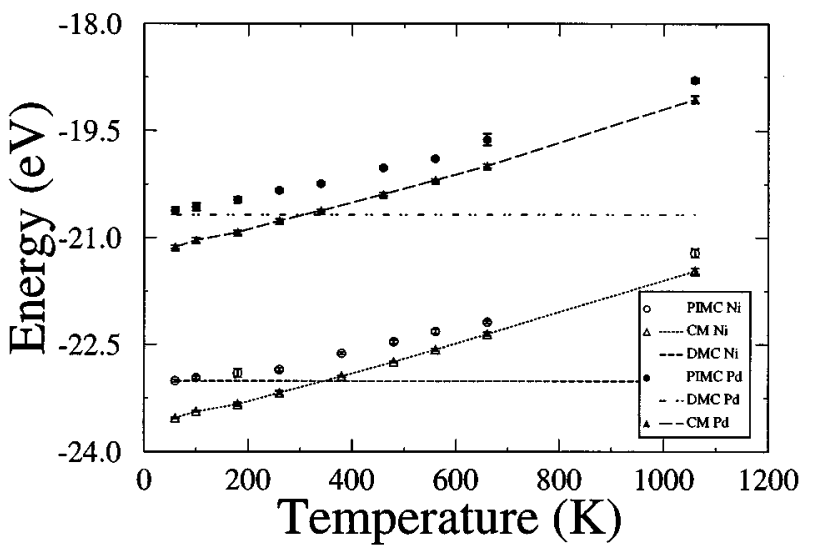

FIG. 11. $\mathrm{Ni}_{7} \mathrm{H}$ and $\mathrm{Pd}_{7} \mathrm{H}$ average energies as a function of temperature: open circle, open triangle and dashed line are the results of PIMC, CM and DMC respectively for the $\mathrm{Ni}_{7} \mathrm{H}$ cluster. Solid circle, solid triangle and dot-dashed line are the results of PIMC, CM and DMC, respectively, for the $\mathrm{Pd}_{7} \mathrm{H}$ cluster.

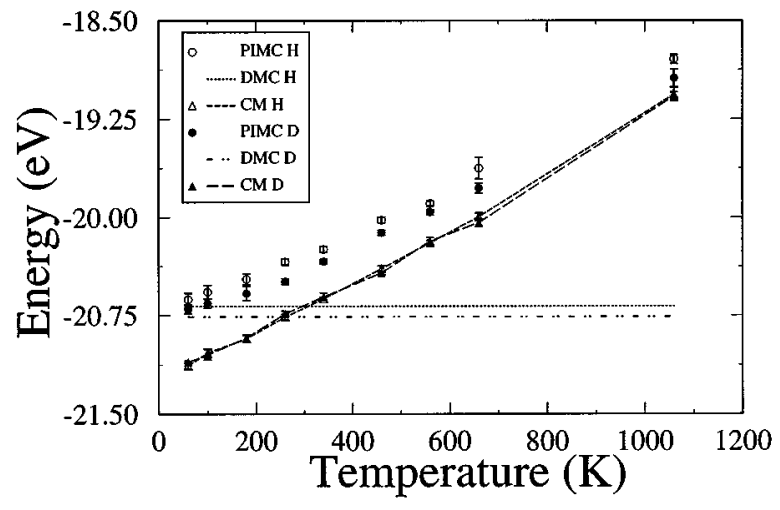

FIG. 12. $\mathrm{Pd}_{7} \mathrm{H}$ and $\mathrm{Pd}_{7} \mathrm{D}$ average energies as a function of temperature: open circle, open triangle and dotted line are the results of PIMC, CM and DMC respectively for the $\mathrm{Pd}_{7} \mathrm{H}$ cluster. Solid circle, solid triangle and dashed line are the results of PIMC, CM and DMC, respectively, for the $\mathrm{Pd}_{7} \mathrm{D}$ cluster.

confinement in ground state. We can therefore roughly estimate that the gap between ground state and the lowest excited state is about $100 \mathrm{~cm}^{-1}$. Because of the highly quantum-mechanical nature of those two clusters $\left(\mathrm{Ni}_{2} \mathrm{H}\right.$ normal mode frequences are $450 \mathrm{~cm}^{-1}, 3022 \mathrm{~cm}^{-1}$ and 4302 $\mathrm{cm}^{-1}, \mathrm{Pd}_{2} \mathrm{H}$ normal mode frequencies are $246 \mathrm{~cm}^{-1}, 2864$ $\mathrm{cm}^{-1}$ and $4214 \mathrm{~cm}^{-1}$ ), the approach to the classical limit with increasing temperature is rather slow.

As the size of the cluster increases, the system becomes less quantum mechanical and the energy gap between ground and first excited state becomes smaller. The calculated curve presented in Fig. 11 does not show a plateau. The absence of a plateau indicates that the first excitation occurs below 60 $\mathrm{K}$. A much faster approach of the PIMC result to the classical limit can be seen from the figure as compared with that of smaller clusters. Figure 12 presents the comparison between $\mathrm{Pd}_{7} \mathrm{H}$ and $\mathrm{Pd}_{7} \mathrm{D}$ clusters. The difference in the quantum calculation between these two isotopes is a result of the difference of the quantum contribution from $\mathrm{H}$ and D. No abnormal isotope effects are observed.

\section{CONCLUSION}

The present paper has examined the properties of $\mathrm{Ni}_{n} \mathrm{Hi}$ and $\mathrm{Pd}_{n} \mathrm{H}$ clusters with $n \leqslant 10$ using a variety of methods. For the optimum cluster geometry, tetrahedral sites are found to be the binding sites for $\mathrm{H}$ for $n \leqslant 5$, whereas octahedral sites are the preferred sites for $n \geqslant 6$. The exception to this rule are $\mathrm{Ni}_{9} \mathrm{H}$ and $\mathrm{Pd}_{9} \mathrm{H}$ for which the outside three-fold hollow and inside tetrahedral sites are the preferred binding sites, respectively. Hydrogen appears to prefer larger binding sites, a result that leads to hydrogen induced rearrangement in $\mathrm{Ni}_{10} \mathrm{H}$ clusters. Because of differences in metal-metal bond lengths, no analogous rearrangement is found in palladium clusters.

DMC calculations show that hydrogen is localized in the present systems. This is consistent with previous surface calculations modeled with the same EAM potential. 
DMC ground state structures are similar to the global potential energy minima with the exceptions of $\mathrm{Pd}_{7} \mathrm{H}$ and $\mathrm{Pd}_{10} \mathrm{H}$. These exceptions reflect the role of both the potential and its curvature in influencing structure.

Finally, finite temperature effects are examined by numerical path integral methods. Quantum contributions from the metal atoms are found to be non-negligable for the total energies, representing up to $1 \%$ of the total energy for seven-metal-atom clusters. Path integral calculations confirm that cluster isomerization is an activated process. The activated nature of the isomerization suggests that care must be exercised to assure proper low temperature sampling. ${ }^{22}$

\section{ACKNOWLEDGMENTS}

We would like to thank D. G. Vlachos for supplying the energies of bare nickel clusters for comparison. We would also like to acknowledge the helpful comments of Frederick G. Haibach. Glman by Daniel Faken was very useful in visualizing cluster structures. This material is based upon work supported under NSF Grant No. CHE9411000, a National Science Foundation and a Cooperative Research Fellowship Program AT\&T graduate fellowship. Research sponsored in part by the Phillips Laboratory, Air Force Material Command, USAF, through the use of Maui High Performance Computing Center (MHPCC) under cooperative agreement number F29601-93-2-0001. The views and conclusions contained in this document are those of the authors and should not be interpreted as necessarily representing the official policies or endorsements, either expressed or implied, of Phillips Laboratory or the U.S. Government.
${ }^{1}$ J. Völkl and G. Alefeld, Diffusion in Solids: Recent Developments, edited by A. S. Nowick and J. J. Burton (Academic, New York, 1975), Chap. 5.

${ }^{2}$ S. W. Rick, D. L. Lynch, and J. D. Doll, J. Chem. Phys. 99, 8183 (1993).

${ }^{3}$ D. L. Lynch, S. W. Rick, M. A. Gomez, B. W. Spath, J. D. Doll, and L. R. Pratt, J. Chem. Phys. 97, 5177 (1992).

${ }^{4}$ K. J. Maynard, A. D. Johnson, S. P. Daley, and S. T. Ceyer, Faraday Discuss. Chem. Soc. 91, 437 (1991).

${ }^{5}$ S. W. Rick, and J. D. Doll, Surf. Sci. Lett. 302, L305 (1994).

${ }^{6}$ M. S. Daw, and M. I. Baskes, Phys. Rev. B. 29, 6443 (1984).

${ }^{7}$ S. E. Wonchoba and D. G. Truhlar, Phys. Rev. B 53, 11222 (1996).

${ }^{8}$ B. M. Rice, B. C. Garrett, M. L. Koszykowski, S. M. Foiles, and M. S. Daw, J. Chem. Phys. 92, 775 (1990).

${ }^{9}$ E. Clementi and C. Roetti, Atomic Data and Nuclear Data Tables (Academic, New York, 1974), Vol. 14, Nos. 3 and 4.

${ }^{10}$ D. G. Vlachos, L. D. Schmidt, and R. Aris, J. Chem. Phys. 96, 6880 (1992).

${ }^{11}$ M. S. Stave and A. E. DePristo, J. Chem. Phys. 97, 3386 (1992).

${ }^{12}$ L. Lian, C.-X. Su, and P. B. Armentrout, J. Chem. Phys. 96, 7542 (1992).

${ }^{13}$ E. K. Parks, L. Zhu, J. Ho, and S. J. Riley, J. Chem. Phys. 100, 7206 (1994).

${ }^{14}$ S. M. Foiles, Surf. Sci. 191, L779 (1987); S. M. Foiles, M. I. Baskes, and M. S. Daw, Phys. Rev. B 33, 7983 (1986).

${ }^{15}$ A. Sachdev and R. I. Masel, J. Mater. Res. 8, 455 (1993).

${ }^{16}$ J. M. Pénisson and A. Renou, J. Cryst. Growth 102, 585 (1990).

${ }^{17}$ W. H. Press, S. A. Teukolsky, W. T. Vetterling, and B. P. Flannery, Numerical Recipies in FORTRAN. The Art of Scientific Computing, 2nd ed. (Cambridge University Press, Cambridge, 1992).

${ }^{18}$ Hydrogen in Metals I \& II, edited by G. Alefeld and J. Völkl (Springer, Berlin, 1978).

${ }^{19}$ D. Ceperley and B. Alder, Science 231, 555 (1986).

${ }^{20}$ P. J. Reynolds, D. M. Ceperley, B. J. Alder, and W. A. Lester, Jr., J. Chem. Phys. 77, 5593 (1982).

${ }^{21}$ J. D. Doll, D. L. Freeman, and T. L. Beck, Adv. Chem. Phys. 78, 61 (1990)

${ }^{22}$ D. D. Frantz, D. L. Freeman, and J. D. Doll, J. Chem. Phys. 93, 2769(1990). 
The Journal of Chemical Physics is copyrighted by the American Institute of Physics (AIP). Redistribution of journal material is subject to the AIP online journal license and/or AIP copyright. For more information, see http:/ojps.aip.org/jcpo/jcpcr/jsp Copyright of Journal of Chemical Physics is the property of American Institute of Physics and its content may not be copied or emailed to multiple sites or posted to a listserv without the copyright holder's express written permission. However, users may print, download, or email articles for individual use. 
The Journal of Chemical Physics is copyrighted by the American Institute of Physics (AIP). Redistribution of journal material is subject to the AIP online journal license and/or AIP copyright. For more information, see http://ojps.aip.org/jcpo/jcper/jsp 